\title{
Ganoderic Acid A-Mediated Modulation of Microglial Polarization is Involved in Depressive-Like Behaviors and Neuroinflammation in a Rat Model of Post-Stroke Depression
}

\author{
Ling Zhang' \\ Lei Zhang ${ }^{2}$ \\ Rubo Sui ${ }^{1}$ \\ 'Department of Neurology, The First \\ Affiliated Hospital of Jinzhou Medical \\ University, Jinzhou, People's Republic of \\ China; ${ }^{2}$ College of Nursing, Jinzhou \\ Medical University, Jinzhou, People's \\ Republic of China
}

Background: Post-stroke depression (PSD) is a common complication after stroke. Ganoderic acid A (GAA), one of the main bioactive Ganoderma triterpenoids, exerts preventive and therapeutic effects in many diseases. However, the function of GAA in PSD has not been well studied.

Methods: PSD model was established via stimulating rats with chronic unpredictable mild stress stimulations (CUMS) after middle cerebral artery occlusion (MCAO). Rats were treated with GAA before CUMS. Depressive-like behaviors were investigated by body weight alteration, open field test (OFT), and sucrose preference test (SPT). Neuronal damage was evaluated by hematoxylin and eosin (HE) staining and Western blotting. Inflammation was detected by enzyme-linked immunosorbent assay (ELISA) and quantitative reverse transcriptase polymerase chain reaction (qRT-PCR). Microglial polarization was analyzed via qRT-PCR and Western blotting. The extracellular signal-regulated kinase (ERK)/cAMPresponse element-binding protein (CREB) pathway was analyzed by Western blotting, and inactivated by the inhibitor PD98059 (PD).

Results: GAA attenuated PSD-induced depressive-like behaviors in rats. GAA mitigated PSD-induced neuronal damage and reduced BDNF and NGF levels in the cerebral hippocampus. GAA weakened PSD-induced inflammatory response in the cerebral hippocampus. GAA prevented pro-inflammatory (M1) polarization and promoted anti-inflammatory (M2) polarization, as indicated by decreased iNOS and CD86 levels and increased Arg-1 and CD206 levels. GAA restored the PSD-induced inactivation of the ERK/CREB pathway. GAA regulated M1/M2 microglial polarization by activating the ERK/CREB pathway.

Conclusion: GAA alleviated the depressive-like behaviors and brain inflammation in PSD rats, indicating its potential for PSD therapy.

Keywords: ganoderic acid A, post-stroke depression, neuronal damage, inflammation, microglial polarization, extracellular signal-regulated kinase

\section{Introduction}

Post-stroke depression (PSD) is a neuropsychiatric disease complicated by stroke, resulting in disability and mortality. ${ }^{1,2}$ The PSD occurs in $45.79 \%$ (34.21\% early and $1.58 \%$ late-onset PSD) of stroke patients in China. ${ }^{3}$ Medeiros et $\mathrm{al}^{4}$ proved that the rates of PSD range from $18 \%$ to $33 \%$, yet it is vastly underdiagnosed and undertreated. Guo et $\mathrm{al}^{5}$ found, based on a large number of studies, that the
College of Nursing, Jinzhou Medical

University, No. 40, Section 3, Songpo

Road, Guta District, Jinzhou, I2100I,

Liaoning Province, People's Republic of

China

Email zhanglei78I27I3@I63.com 
incidence of PSD within 2 years was $11 \%$ to $41 \%$. In the last few years, many reports have studied antidepressants, while the effective treatments for PSD remain limited. ${ }^{6}$ The PSD is related to the neuronal damage, inflammation, and microglial polarization. ${ }^{7-9}$ Hence, exploring new drugs targeting these events may help to find promising approaches for PSD therapy.

Traditional Chinese medicine is a common therapy for depression. ${ }^{10}$ Ganoderic acid A (GAA), one of the major triterpenoids isolated from Ganoderma, is a traditional Chinese medicine and plays anti-oxidant, anti-inflammatory, and anti-tumor activities. ${ }^{11-13}$ A pharmacokinetic research of GAA in rats shows that GAA can rapidly absorb in the circulation after orally dosing and can enter the brain lateral ventricle after intravenously dosing with an $8.68 \%$ or $2.96 \%$ of bioavailability, respectively. ${ }^{14}$ Moreover, GAA protects against neuronal damage under nitric oxide stress or epilepsy. ${ }^{15,16}$ Thus, we hypothesize that GAA has a potential neuroprotective function. However, the function of GAA in PSD is largely unknown.

The purposes of this research were to study the pharmacological effects of GAA on PSD in rat model and to explore its potential mechanism. This study might provide a new therapy strategy for PSD.

\section{Materials and Methods}

\section{Animal Experiments}

The Sprague-Dawley rats (male; 240-260 g) were provided by Sipeifu (Beijing, China) and maintained in a specific environment. A total of 62 rats were used in this study. Eight rats were in the sham group. Fifty-four rats underwent middle cerebral artery occlusion (MCAO) surgery. Of these, $4 \mathrm{MCAO}$ rats died within 2 days post MCAO surgery. Two survivors that did not meet neurological functional score were excluded from the present study. The surviving MCAO rats were randomly assigned to 6 groups. Three rats died before the sacrifice and acquisition of brain tissue. The final numbers of rats in each group were 8 (sham), 7 (MCAO), 7 (PSD), 8 (PSD+L-GAA), 8 (PSD+M-GAA), 8 (PSD+H-GAA), and 7 (PSD+PD+GAA), respectively. (1) Rats in the sham group were not subjected to stress but the sham operation. (2) the rats in the MCAO group were introduced with a nylon to the ends via the right internal carotid to the right middle cerebral artery. ${ }^{17}$ (3) the rats in the PSD group were exposed to CUMS for 3 weeks. Rats in the PSD group were intravenously injected with normal saline. (4) Rats in the PSD + L-GAA group were intravenously injected with $10 \mathrm{mg} / \mathrm{mL}$ GAA (MedChemExpress, Monmouth Junction, NJ, USA) before PSD. (5) Rats in the PSD + M-GAA group were intravenously injected with $20 \mathrm{mg} / \mathrm{mL}$ GAA before PSD. (6) Rats in the PSD + H-GAA group were intravenously injected with $30 \mathrm{mg} / \mathrm{mL}$ GAA before PSD. (7) Rats in the PSD + PD + GAA group were treated with $5 \mu \mathrm{L} 100$ $\mu \mathrm{M}$ of PD98059 (PD; MedChemExpress) by intracerebroventricular injection and intravenously injected with $30 \mathrm{mg} / \mathrm{mL}$ GAA before PSD. CUMS was conducted 2 days after MCAO as previously described. ${ }^{2}$ Briefly, the CUMS was composed of 7 different stimulating factors, including: stimulation with $80 \mathrm{~dB}$ noise for $12 \mathrm{~h}$; tilting the cage $\sim 45^{\circ}$ in moist bedding for $24 \mathrm{~h}$; stimulation with LED flash for $12 \mathrm{~h}$; swimming in ice water for 5 min; food and water deprivation for $12 \mathrm{~h}$; reversing the light of day and night; and clamping the tail with the needle holder for $3 \mathrm{~min}$. Rats were received one random stimulus daily, and the stressors were conducted for 3 weeks. Body weight and behaviors were measured after MCAO and CUMS weekly. Then, rats were sacrificed by cervical dislocation, and the hippocampus tissues were collected for histopathological or molecular analysis. Animal experiments were performed in keeping with the Guide for the Care and Use of Laboratory Animals $^{18}$ and permitted by the Animal Ethical Committee of The First Affiliated Hospital of Jinzhou Medical University (approval No. IACUC 2019007).

\section{Neurological Functional Assessments}

Neurological functions of MCAO rats were determined using a modified Longa five-point scale scoring system. Evaluators were blinded to the experimental groups. The five-point scale was as follows: 0: no neurological deficits; 1: failure to extend the contralateral forepaw fully when held by the tail; 2: spontaneous left circling; 3 : falls to the contralateral side of brain damage; and 4: no spontaneous movement or loss of consciousness. Rats with scores of 13 points were included in the follow-up experiments.

\section{Open Field Test (OFT) and Sucrose Preference Test (SPT)}

The behavioral analyses were conducted by experimenters who were blind to the experimental groups. The behavioral tests included OFT and SPT. The OFT was conducted to test the spontaneous activity and anxiety-like 
behavior as previously reported. ${ }^{19}$ In brief, rats were individually placed in the center of the open-field test box (100 $\times 100 \times 40 \mathrm{~cm}$ ), and allowed to freely explore for $5 \mathrm{~min}$. The behaviors were recorded using the animal behavior video analysis system (Xinxin Soft Information Technology, Shanghai, China). Times of rats' horizontal and vertical activities were analyzed. The OFT scores were recorded after CUMS.

The SPT was performed to investigate the state of anhedonia according to the protocols as a previous report. ${ }^{20}$ Briefly, rats were kept in separate cages with 2 bottles of $1 \%$ sucrose solution the first day. Then, one bottle of the sucrose solution was changed to a bottle of tap water the second day. SPT was carried out the next day. In the test phase, rats were deprived of water and food for $24 \mathrm{~h}$, and then placed in a cage containing one bottle of $1 \%$ sucrose solution and one bottle of tap water for 3 h. The position of the two bottles was changed every hour. The test was performed after CUMS. The consumption of sucrose was recorded.

\section{Hematoxylin and Eosin (HE) Staining}

The hippocampus tissues were collected, and immersed in $3.7 \%$ formaldehyde (Sigma-Aldrich, St. Louis, MO, USA), followed via dehydration and paraffin embedding. After that, the tissues were cut to $4-\mu \mathrm{m}$ sections. Next, the sections were deparaffinized, rehydrated, and stained with hematoxylin and eosin (Beyotime, Shanghai, China) for 10 min. The images of hippocampus sections were observed under a microscope (Olympus, Tokyo, Japan).

\section{Western Blotting}

Hippocampus tissues were lysed in radioimmunoprecipitation assay buffer (Beyotime) containing $1 \%$ protease/phosphatase inhibitor (Beyotime). The protein samples were harvested after centrifugation at 10,000 $\times \mathrm{g}$ for $5 \mathrm{~min}$, and quantified with a bicinchoninic acid (BCA) assay kit (Beyotime). The samples (20 $\mu \mathrm{g})$ were separated via sodium dodecyl sulfate-polyacrylamide gel electrophoresis, and transferred onto polyvinylidene fluoride membranes (Bio-Rad, Hercules, CA, USA). After blocking in 3\% bovine serum albumin (Solarbio, Beijing, China), the membrane was incubated with anti-BDNF (ab108319, 1:1000 dilution, Abcam), anti-NGF (ab52918, 1:500 dilution, Abcam), anti-iNOS (ab136918, 1:2000 dilution, Abcam), anti-CD86 (ab112490, 1:500 dilution, Abcam), anti-Arg-1 (ab203490, 1:1000 dilution, Abcam), anti-CD206 (ab64693, 1:500 dilution, Abcam), anti-ERK (ab184699, 1:1000 dilution, Abcam), anti-p-ERK (ab201015, 1:500 dilution, Abcam), anti-CREB (ab32515, 1:500 dilution, Abcam), anti-p-CREB (ab32096, 1:1000 dilution, Abcam), or anti- $\beta$-actin (ab20272, 1:5000 dilution, Abcam) antibody overnight at $4^{\circ} \mathrm{C}$. Membranes were then incubated with HRPconjugated IgG (ab6721, 1:5000 dilution, Abcam) for 2 h. $\beta$-actin acted as a control. After exposure to the ECL Western Blotting Substrate (Solarbio), the blots were analyzed utilizing Quantity One software (Bio-Rad).

\section{Enzyme-Linked Immunosorbent Assay (ELISA)}

The hippocampus samples of rats were used to detect inflammatory cytokines using the TNF- $\alpha$, IL-1 $\beta$, IL-6, or IL-10 ELISA kit (Thermo Fisher Scientific, Waltham, MA, USA) in accordance with the kit's instructions. The absorption at $450 \mathrm{~nm}$ was measured through a microplate reader (Biotek, Winooski, VT, USA). The concentration of inflammatory cytokine was calculated according to the standard curve. Total protein concentration was determined with a BCA assay kit. The levels of inflammatory cytokines were expressed as the calculated concentration/total protein.

\section{Quantitative Reverse Transcriptase Polymerase Chain Reaction (qRT-PCR)}

Hippocampus tissues were lysed in Trizol (Vazyme, Nanjing, China), and RNA was isolated according to the protocols. The cDNA was generated from 800 ng RNA using M-MLV reverse transcription kit (Thermo Fisher Scientific), and used for qRT-PCR together with SYBR (Thermo Fisher Scientific) and primer pairs. The specific primers were designed by GenScript (Nanjing, China), and the sequences were shown as follows: TNF- $\alpha$ (Forward: 5'-ACTGAACTTCG GGGTGATCG-3', Reverse: 5'-GCTTGGTG GTTTGCTACGAC-3'); IL-1 $\beta$ (Forward: 5'-TTGA GTCTGCACAGTTCCCC-3'， Reverse: 5'-GTCCTGGG GAAGGCATTAGG-3'); IL-6 (Forward: 5'-CCAGTTGCCT TCTTGGGACT-3', Reverse: 5'-TCTGACAGTGCAT CATCGCT-3'); IL-10 (Forward: 5'-CCTGCTCTTACTG GCTGGAG-3', Reverse: 5'-TGTTGTCCAGCTG GTCCTTC-3'); iNOS (Forward: 5'-GGTGAGGGGA CTGGACTTTTAG-3', Reverse: 5'-TTGTTGGGCTGGGA ATAGCA-3'); CD86 (Forward: 5'-CGAACACTATTT GGGCGCAG-3', Reverse: 5'-CAAACTGGGGCTGCG AAAAA-3'); Arg-1 (Forward: 5'-GGACATCGTGTACA TCGGCT-3', Reverse: 5'-TTTGCTGTGATGCCCCAGAT 
-3'); CD206 (Forward: 5'-GTGCAAACACTG

GGCAGAAG-3', Reverse: 5'-ATTGTCTTGAGGA GCGGGTG-3'); and GAPDH (Forward: 5' -GAAGG TCGGTGTGAACGGAT-3', Reverse: 5 ' -GGCATGGA CTGTGGTCATGA-3'). GAPDH functioned as a reference. Relative RNA expression was calculated with $2^{-\Delta \Delta \mathrm{Ct}}$ method.

\section{Statistical Analysis}

The data were shown as mean \pm standard deviation from 3 repeats. The difference was investigated by analysis of variance (ANOVA) followed via Tukey post hoc test using GraphPad Prism 8 (GraphPad, La Jolla, CA, USA). It was significant at $P<0.05$.

\section{Results}

\section{GAA Relieves PSD-Induced}

\section{Depressive-Like Behaviors}

To study the function of GAA in PSD, we firstly established PSD model through stimulating rats with 3-week CUMS after MCAO treatment, and rats were treated with different concentrations of GAA prior to CUMS stimulation (Figure 1A). The rats were divided into sham, MCAO, PSD, PSD + L-GAA, PSD+ M-GAA, or PSD + H-GAA group. The structural formula of GAA is displayed in Figure 1B. Moreover, the body weight of rats was detected in each group. As shown in Figure 1C, the body weight of rats in the PSD group was markedly declined in comparison to the sham group, but administration of GAA attenuated the weight loss compared with the PSD group, especially in the PSD+ M-GAA or PSD + H-GAA groups. Additionally, the depressive-like behaviors were assessed with OFT and SPT. After 3 weeks of CUMS, a significant decrease in motion activity and sucrose consumption was observed in the PSD group relative to the sham group, which was stored after GAA administration (Figure 1D-F). Besides, there was no significant difference in the results between the PSD group and the PSD+Vehicle group (Supplemental Figure 1). These results indicated that GAA mitigated the depressive-like behaviors in PSD rats.

\section{GAA Weakens PSD-Induced Neuronal Damage in Cerebral Hippocampus of PSD Rats}

To explore the effect of GAA on neuronal damage in PSD rats, HE staining was performed to detect the morphological change in the hippocampus. As exhibited in Figure 2A, the neurons were clearly impaired, sparse, and absent in the hippocampal CA1 region in the PSD group relative to the sham group, but this neuronal damage was progressively weakened by GAA treatment. Furthermore, the BDNF and NGF proteins were closely linked to the hippocampal neuronal damage. Hence, their expression levels were detected in the hippocampus. BDNF and NGF protein levels were significantly reduced after MCAO treatment, and their levels were further downregulated by PSD stimulation, while GAA revised this effect (Figure 2B-D). These findings suggested that GAA attenuated the neuronal damage in PSD rats.
A

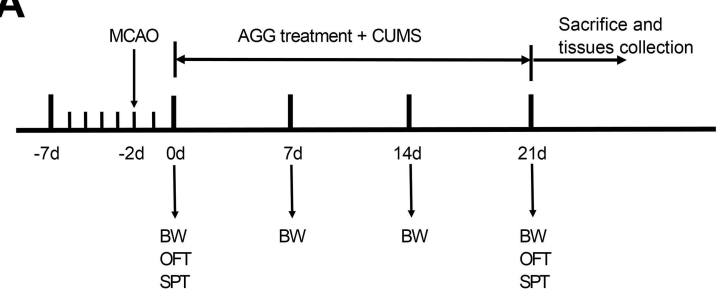

B

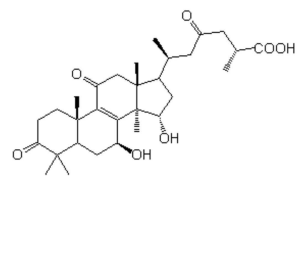

C

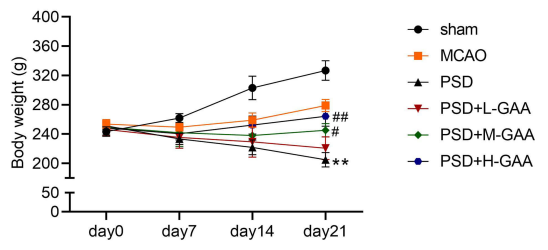

D

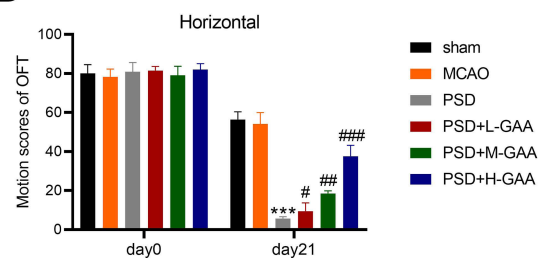

E

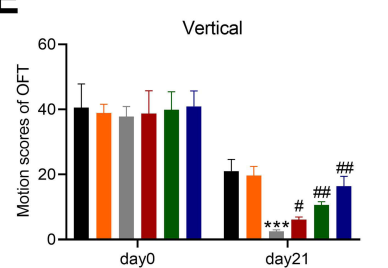

$\mathbf{F}$

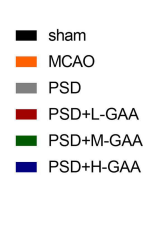

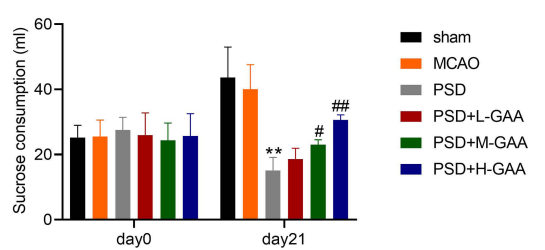

Figure I Effect of GAA on PSD-induced depressive-like behaviors in rats. (A) Experimental timeline. (B) The structural formula of GAA. (C) Body weight in each group. ( $D$ and $\mathbf{E}$ ) The horizontal and vertical motion scores of open field test (OFT) in each group. (F) Sucrose preference test (SPT) consumption in each group. $n=8$. MCAO: stroke model; PSD: CUMS after MCAO; L-GAA: low dose (10 mg/kg) of GAA; M-GAA: median dose (20 mg/kg) of GAA; H-GAA: high dose (30 mg/kg) of GAA. **P<0.0I and $* * * P<0.001$ compared with $M C A O$ group; ${ }^{\#} P<0.05,{ }^{\#} P<0.01$, and ${ }^{\# \#} P<0.001$ compared with PSD group. 


\section{GAA Alleviates PSD-Induced Inflammation in Cerebral Hippocampus of PSD Rats}

To analyze the effect of GAA on the inflammation in PSD rats, the related inflammatory cytokine levels in the hippocampus were detected. As shown in Figure 3A-D, TNF- $\alpha$, IL-1 $\beta$, and IL-6 levels in hippocampus were obviously elevated, and IL-10 level was markedly decreased in the MCAO and PSD groups compared with the sham group, and there were significant differences in levels of IL- $1 \beta$ and IL-10 between the PSD and MCAO groups. However, GAA administration reversed this influence (Figure 3AD). In addition, these inflammatory cytokine levels were measured in hippocampus tissues. TNF- $\alpha$, IL- $1 \beta$, and IL- 6 expression levels were markedly upregulated, and IL-10 expression was obviously downregulated in the MCAO group compared with the sham group (Figure 3E-H). Moreover, higher levels of TNF- $\alpha$, IL-1 $\beta$, and IL-6, and lower expression of IL-10 were observed in the PSD group than those in the MCAO group (Figure 3E-H). However, GAA administration relieved the promotion of TNF- $\alpha$, IL$1 \beta$, and IL- 6 , and inhibition of IL-10 after PSD stimulation (Figure 3E-H). These data suggested that GAA weakened the inflammation in PSD rats.

\section{GAA Regulates Microglial Phenotypic Profiles and Polarization in Cerebral Hippocampus of PSD Rats}

To analyze the function of GAA in M1/M2 microglial polarization in PSD rats, the expression levels of M1/M2 microglial markers were measured in hippocampus tissues. The levels of M1 microglial markers (iNOS and CD86) were clearly upregulated in the MCAO and PSD groups relative to the sham group, and they were higher in the PSD group than the MCAO group. GAA addition progressively decreased their expression compared with the PSD group (Figure 4A-C). Furthermore, the levels of M2 microglial markers (Arg-1 and CD206) were greatly downregulated in the MCAO and PSD groups compared with the sham group, and they were lower in the PSD group than the MCAO group. However, GAA treatment restored their levels relative to the PSD group (Figure 4DF). These results indicated that GAA promoted M1/M2 microglial polarization in PSD rats.

\section{GAA Reverses PSD-Induced Inactivation of the ERK/CREB Pathway}

To probe the potential mechanism involved in the function of GAA in PSD rats, the levels of protein related to the

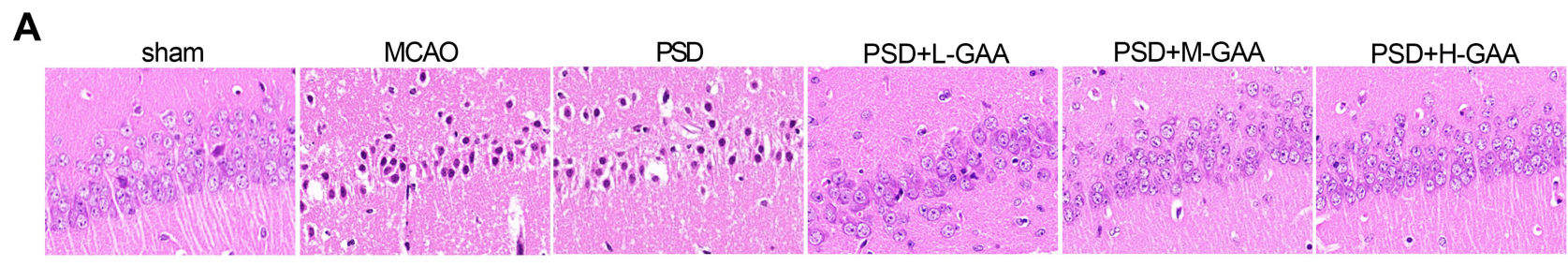

B

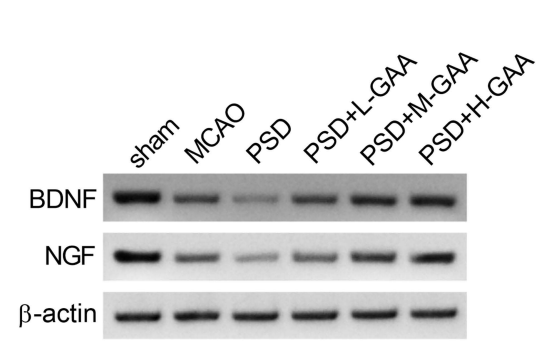

C

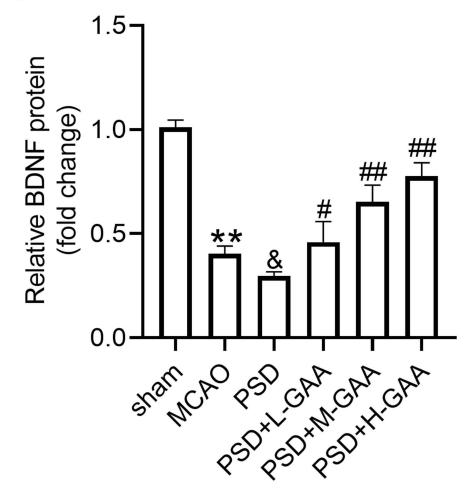

D

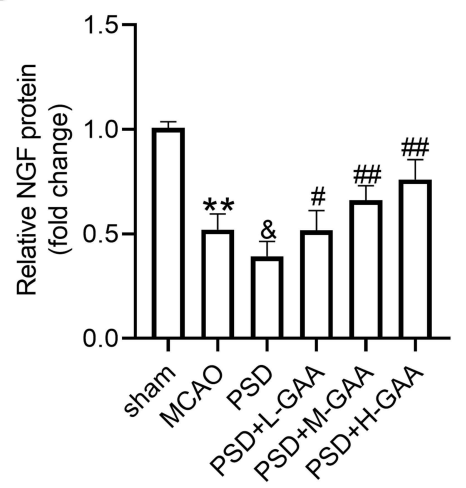

Figure 2 Effect of GAA on PSD-induced neuronal damage in the hippocampus. (A) The histological analysis of hippocampus by HE staining. (B-D) Western blotting for protein levels of BDNF and NGF in hippocampus tissues. $n=8$. MCAO: stroke model; PSD: CUMS after MCAO; L-GAA: low dose (I0 mg/kg) of GAA; M-GAA: median dose $(20 \mathrm{mg} / \mathrm{kg})$ of GAA; H-GAA: high dose $(30 \mathrm{mg} / \mathrm{kg})$ of GAA. ${ }^{*} P\left(P<0.01\right.$ compared with the sham group; ${ }^{\circledR} P<0.05$ compared with the MCAO group; ${ }^{\#} P<0.05$ and ${ }^{\# \#} P<0.01$ compared with the PSD group. 
A

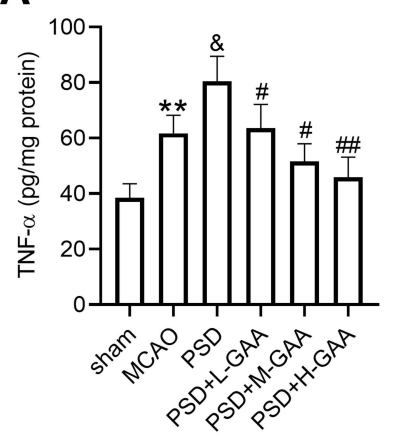

E

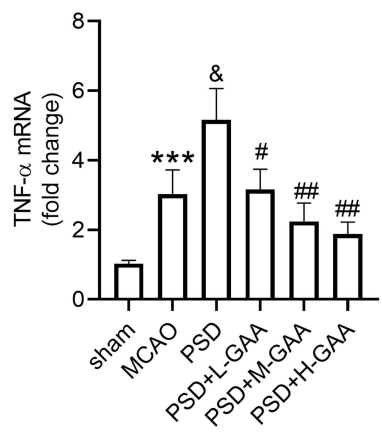

B

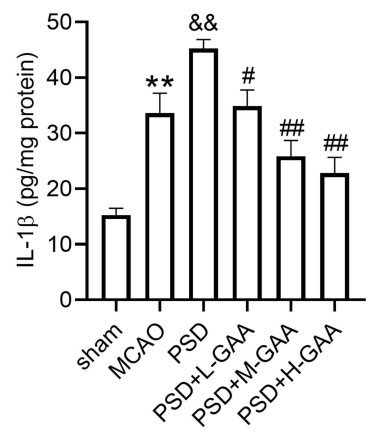

F

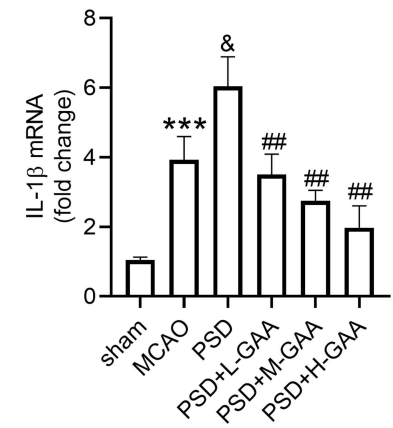

C

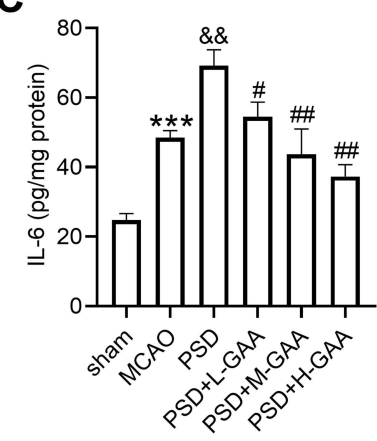

G

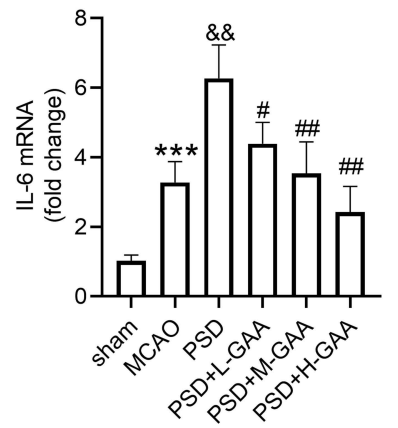

D

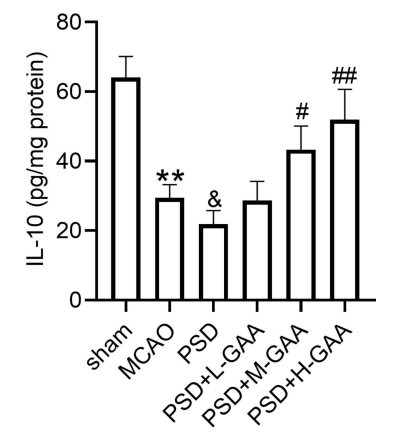

H

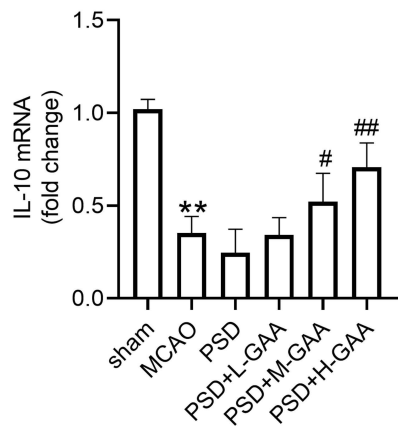

Figure 3 Influence of GAA on inflammatory cytokine expression in PSD rats. (A-D) ELISA assay of TNF- $\alpha$, IL-I $\beta$, IL-6, and IL-I0 in hippocampus. (E-H) qRT-PCR for expression of TNF- $\alpha$, IL-I $\beta$, IL-6, and IL-10 in hippocampus. $n=8$. MCAO: stroke model; PSD: CUMS after MCAO; L-GAA: low dose (I0 mg/kg) of GAA; M-GAA: median dose $(20 \mathrm{mg} / \mathrm{kg})$ of GAA; H-GAA: high dose $(30 \mathrm{mg} / \mathrm{kg})$ of GAA. ${ }^{* * P} P<0.0 \mathrm{I}$ and $* * * P<0.00 \mathrm{I}$ compared with the sham group; ${ }^{\circledR} P<0.05$ and ${ }^{\& \&} P<0.0 \mathrm{I}$ compared with the MCAO group; ${ }^{\#} P<0.05$ and ${ }^{\#} P<0.01$ compared with the PSD group.

ERK/CREB signaling were detected in hippocampus tissues. As displayed in Figure 5A-C, the levels of p-ERK/ ERK and $\mathrm{p}-\mathrm{CREB} / \mathrm{CREB}$ were significantly reduced after MCAO or PSD treatment, and PSD group showed the lower phosphorylation levels of ERK and CREB, while GAA administration reversed this effect. These results indicated that GAA promoted activation of the ERK/ CREB signaling in PSD rats.

\section{Blockage of the ERK/CREB Signaling Attenuates the Effect of GAA on Microglial Polarization}

To explore whether the ERK/CREB signaling was linked to the function of GAA in microglial polarization in PSD rats, the inhibitor PD was used before CUMS, and the expression levels of microglial markers were examined in hippocampus tissues (Figure 6A). As exhibited in Figure $6 \mathrm{~B}-\mathrm{D}$, PD treatment abolished the suppressive effect of GAA on levels of iNOS and CD86 after PSD stimulation. In addition, the addition of PD reversed GAAinduced upregulation of Arg-1 and CD206 in PSD rats (Figure $6 \mathrm{E}-\mathrm{G}$ ). These data suggested that GAA inhibited
M1 microglial polarization and facilitated M2 microglial polarization by activating the ERK/CREB pathway in PSD rats.

\section{Discussion}

PSD is a frequent mood disorder in stroke patients and a major predictor of poor outcome after stroke. ${ }^{21,22}$ Traditional Chinese medicine can be used to treat depression. ${ }^{23}$ Our study wanted to explore the function of GAA isolated from traditional Chinese medicine Ganoderma in PSD. Here, we firstly found GAA attenuated PSD symptoms in a rat model, indicating the potential of GAA for PSD therapy.

In this study, we established the PSD model through CUMS after stroke induced by MCAO. The depressivelike behaviors were displayed by the reduced motion activity and sucrose consumption, which was consistent with a previous study. ${ }^{19}$ Moreover, we found GAA could prevent these depressive-like behaviors in rats. Furthermore, we performed a histological analysis and found that GAA attenuated the hippocampus tissue damage in PSD rats. Previous reports suggested that 
A

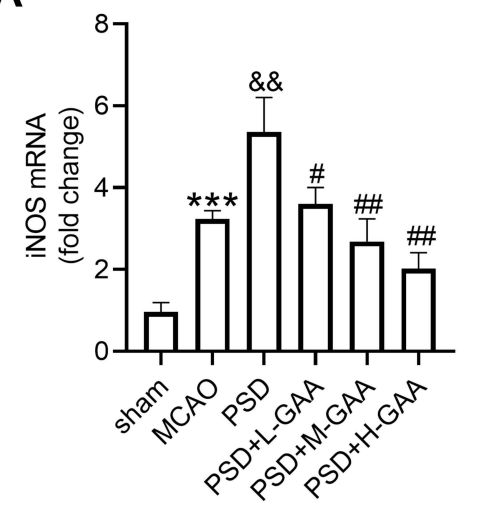

D

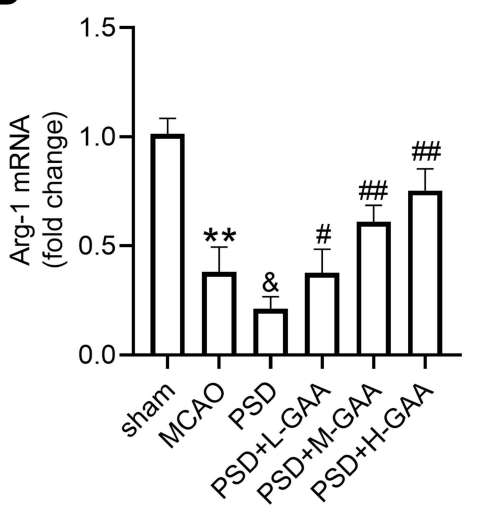

B

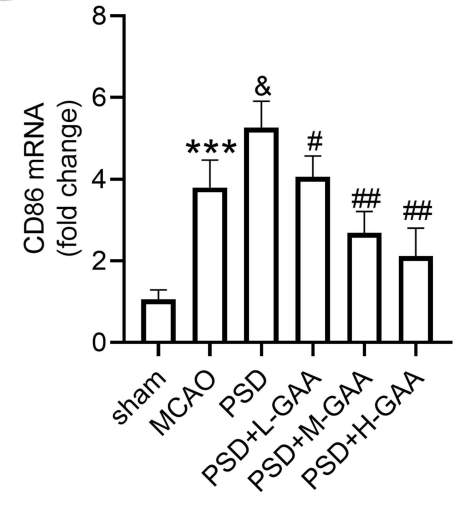

E

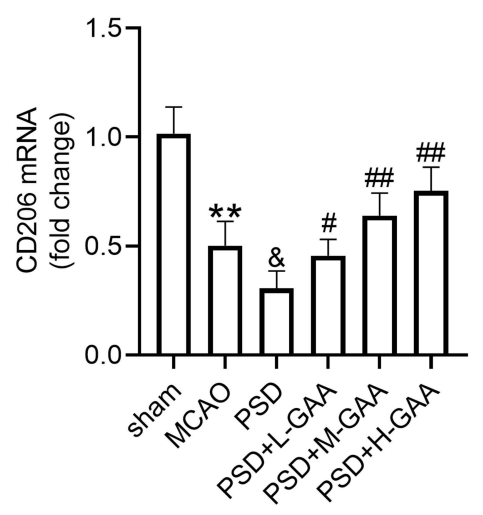

C

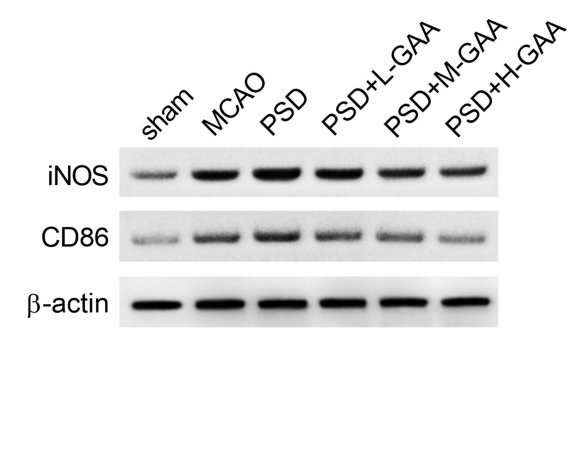

F

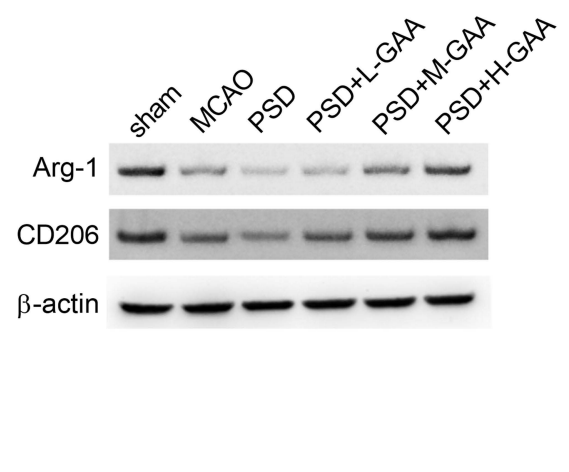

Figure 4 Influence of GAA on microglial phenotypic profiles and polarization. (A-C) qRT-PCR and Western blotting for expression levels of iNOS and CD86 in hippocampus tissues. (D-F) qRT-PCR and Western blotting for expression of Arg-I and CD206 in hippocampus tissues. n=8. MCAO: stroke model; PSD: CUMS after MCAO; L-GAA: low dose ( $10 \mathrm{mg} / \mathrm{kg})$ of GAA; M-GAA: median dose $(20 \mathrm{mg} / \mathrm{kg})$ of GAA; H-GAA: high dose $(30 \mathrm{mg} / \mathrm{kg})$ of GAA. **P<0.0I and $* * * P<0.00 \mathrm{I}$ compared with the sham group; ${ }^{\&} P<0.05$ and ${ }^{\& \&} P<0.01$ compared with the MCAO group; ${ }^{\#} P<0.05$ and ${ }^{\# \#} P<0.01$ compared with the PSD group.

A

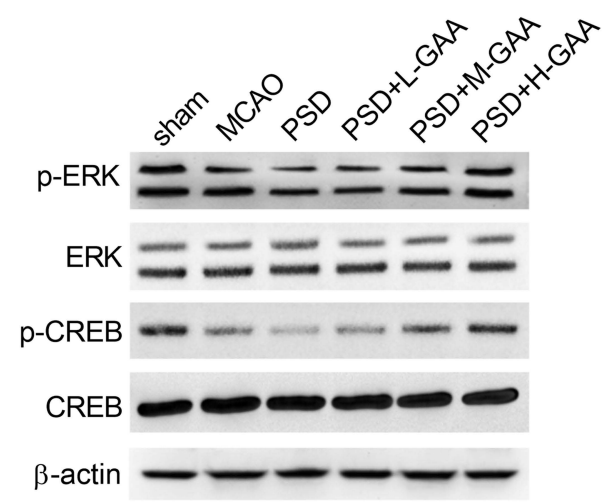

B

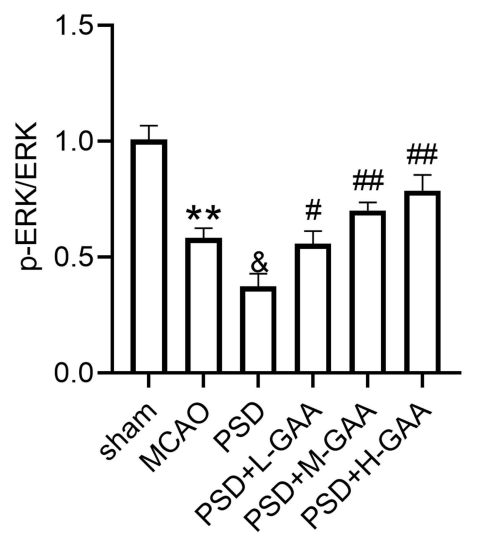

C

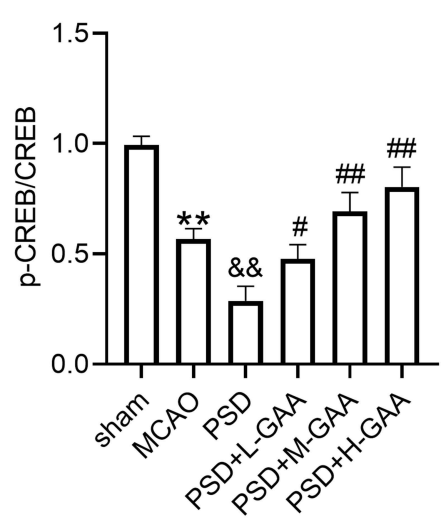

Figure 5 Impact of GAA on activation of the ERK/CREB pathway in PSD rats. (A-C) Western blotting for phosphorylation levels of ERK and CREB in hippocampus tissues. $\mathrm{n}=8$. MCAO: stroke model; PSD: CUMS after MCAO; L-GAA: low dose (10 mg/kg) of GAA; M-GAA: median dose $(20 \mathrm{mg} / \mathrm{kg})$ of GAA; H-GAA: high dose (30 mg/kg) of GAA. ${ }^{* * P} P<0.01$ compared with the sham group; ${ }^{\&} P<0.05$ and ${ }^{\&} \&<<0.01$ compared with the MCAO group; ${ }^{\#} P<0.05$ and ${ }^{\# \#} P<0.01$ compared with the PSD group. 


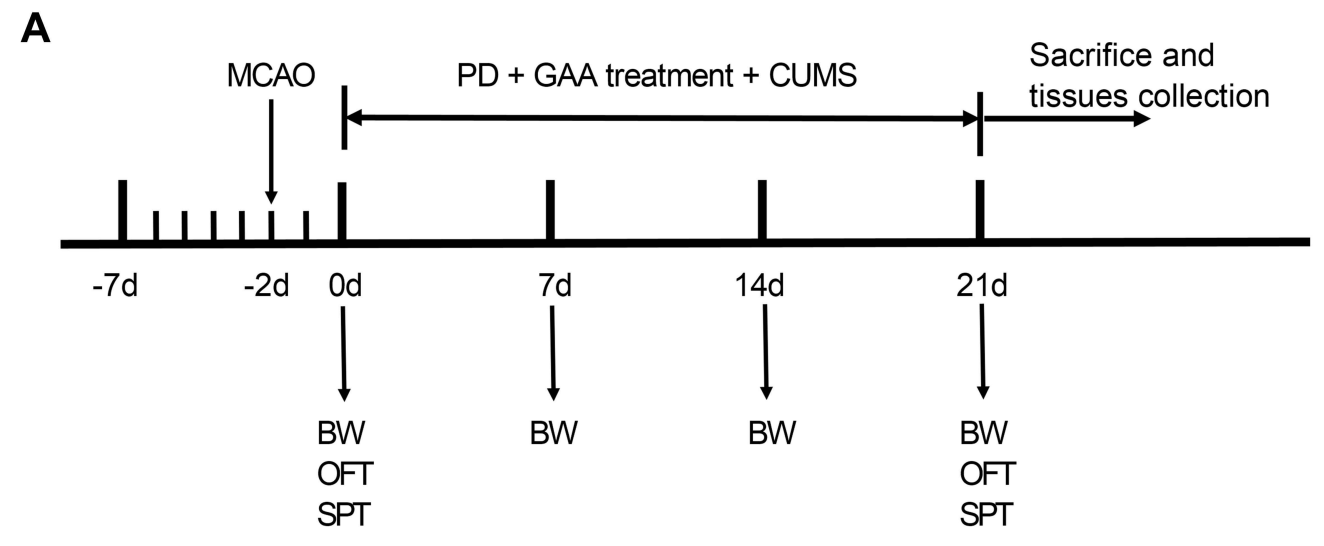

B

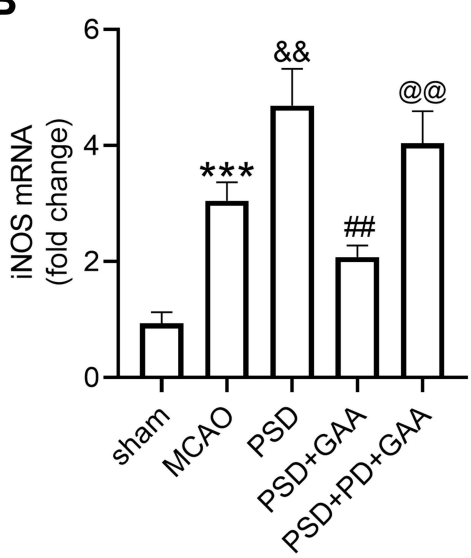

E

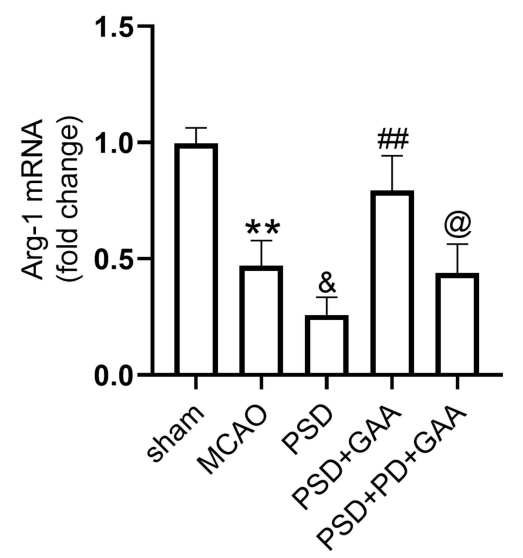

C

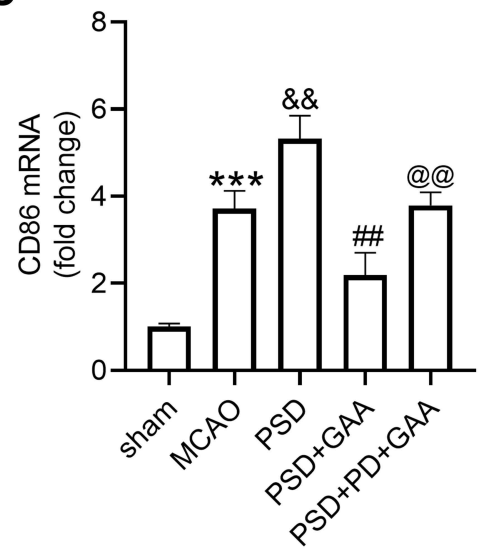

F

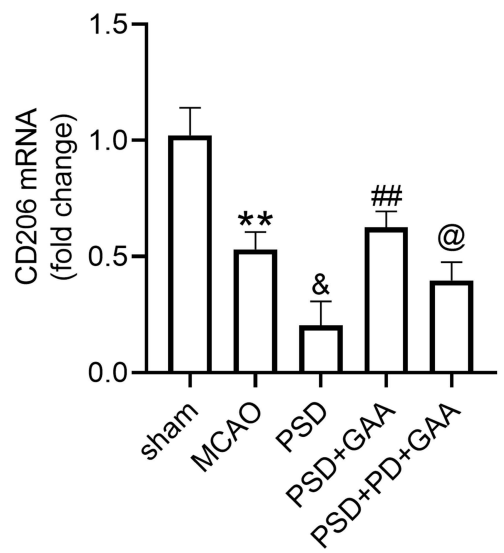

D

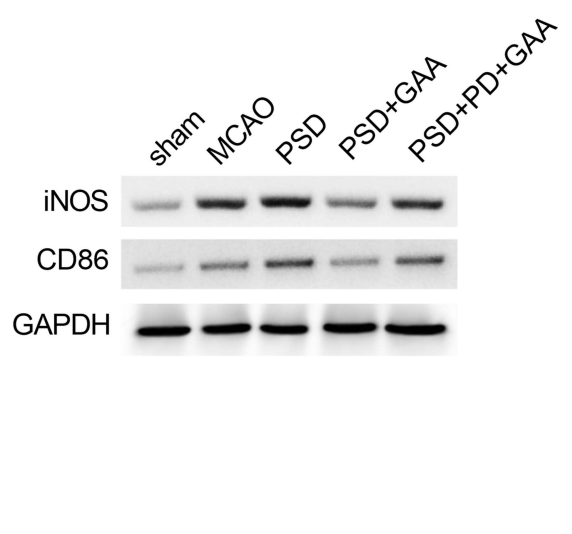

G

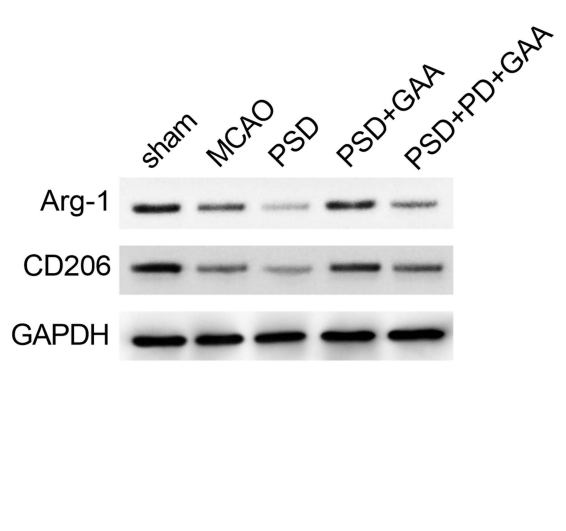

Figure 6 Effect of GAA on MI/M2 microglial polarization depends on the ERK/CREB pathway. (A) Experimental timeline. (B-D) qRT-PCR and Western blotting for expression of iNOS and CD86 in hippocampus tissues. (E-G) qRT-PCR and Western blotting for expression of Arg- 1 and CD206 in hippocampus tissues. $n=8$. MCAO: stroke model; PSD: CUMS after MCAO; GAA: high dose $(30 \mathrm{mg} / \mathrm{kg}$ ) of GAA; PD: $100 \mu \mathrm{M}$ PD (ERK inhibitor). $* * P<0.01$ and $* * * P<0.001$ compared with the sham group; ${ }^{\&} P<0.05$ and ${ }^{\& \&} P<0.01$ compared with the MCAO group; ${ }^{\prime} P<0.01$ compared with the PSD group; ${ }^{@} P<0.05$ and ${ }^{@ @ P} P<0.01$ compared with the PSD+GAA group.

BDNF and NGF were involved in depression, and a reduction in $\mathrm{BDNF}$ and $\mathrm{NGF}$ was responded to CUMS. $^{24,25}$ Upregulation of BDNF and NGF induced the survival of hippocampus neurons. ${ }^{26}$ Hence, we detected BDNF and NGF expression in hippocampus samples, and found that GAA mitigated PSD-induced downregulation, which was consistent to a former work in sclerosis. ${ }^{12}$ Additionally, inflammation is another feature 
of PSD. ${ }^{27}$ The pro-inflammatory cytokines (TNF- $\alpha$, IL-1 $\beta$, and IL-6) and anti-inflammatory cytokine (IL-10) are related to the inflammatory response in depressive disorder. $^{28}$ Here, we confirmed that GAA weakened the inflammatory response in PSD rats by decreasing TNF- $\alpha$, IL-1 $\beta$, and IL-6 and increasing IL-10 level, indicating the anti-inflammatory role of GAA. These results were also in agreement with previous studies on other diseases. ${ }^{12,29,30}$ These findings suggested the therapeutic effect of GAA on PSD rats.

Similar to macrophages, microglia also exhibit polarity, including M1 (pro-inflammatory) and M2 (anti-inflammatory) phenotypes. $^{31}$ Microglia polarization is considered as a new strategy for the treatment of ischemic stroke. ${ }^{32}$ Neuroinflammation plays an important role in the development of depression and is considered a sign of depression. M1/ M2 phenotype microglia acts as critical mediators in the pathological process of neuroinflammation. ${ }^{33}$ In addition, $\mathrm{M} 1 / \mathrm{M} 2$ microglial polarization is associated with psychiatric disorders. ${ }^{9}$ Inhibition of M1 microglial polarization and promotion of M2 microglial polarization contribute to the antidepressant therapies. ${ }^{34,35}$ By detecting the microglial markers, we found that GAA repressed M1 microglial polarization and increased M2 microglial polarization in PSD rats. Considering the important role of microglia M1/M2 polarization in stroke and depression, we believe that the anti-inflammatory effect of GAA in PSD rats may be through inducing microglia to polarize the anti-inflammatory M2 phenotype.

Next, we wanted to explore a potential mechanism driven by GAA. Previous studies have reported that GAA could inhibit the Janus kinase 2/signal transducer and activator of transcription 3, phosphoinositide 3-kinase/protein kinase $\mathrm{B}$, and nuclear factor- $\mathrm{KB}$ pathways in many diseases. ${ }^{13,30,36}$ Here, we aimed to explore a new downstream pathway for GAA. The ERK/CREB pathway, a downstream signaling of BDNF, plays important roles in depressive disorders. ${ }^{37}$ Many studies have reported that this pathway was related to the prevention of depressive-like behaviors. ${ }^{38,39}$ Having established the increased BDNF by GAA, we assumed that the ERK/ CREB pathway was also mediated by GAA. Here, we found that this pathway was inactivated in PSD rats after CUMS, which was also consistent with previous reports. ${ }^{40-42}$ Furthermore, our study showed that GAA could activate the ERK/CREB pathway by increasing the phosphorylation of ERK and CREB. Additionally, the inhibition of this signaling using PD reversed GAAinduced M1/M2 microglial polarization, further suggesting GAA exerted its activity in PSD by regulating the ERK/CREB pathway.

In conclusion, our research showed that GAA prevented the depressive-like behaviors, neuronal damage, inflammation, and M1/M2 microglial polarization in PSD rats. This study reveals a novel insight into the function of GAA in PSD rats, and indicates the potential of GAA for PSD treatment.

\section{Funding}

This paper was funded by Science and Technology Project of Education Department of Liaoning Province (JYTJCZR201903), Basic Research Project of Education Department of Liaoning Province (JYTJCZR2020065), Youth Fund Project of Education Department of Liaoning Province (JYTQN201707), Nature Foundation Guidance Program Project of Liaoning Province (2019-ZD-0802), and National Natural Science Foundation of China (81371461, 81241050).

\section{Disclosure}

The authors declare that they have no conflict of interests.

\section{References}

1. Robinson RG, Jorge RE. Post-stroke depression: a review. Am $J \quad$ Psychiatry. 2016;173(3):221-231. doi:10.1176/appi. ajp.2015.15030363

2. Durocher M, Ander BP, Jickling G, et al. Inflammatory, regulatory, and autophagy co-expression modules and hub genes underlie the peripheral immune response to human intracerebral hemorrhage. J Neuroinflammation. 2019;16(1):1-21. DOI:10.1186/s12974-0191433-4

3. Zhao FY, Yue YY, Li L, et al. Clinical practice guidelines for post-stroke depression in China. Braz J Psychiatry. 2018;40 (3):325-334. doi:10.1590/1516-4446-2017-2343

4. Medeiros GC, Roy D, Kontos N, et al. Post-stroke depression: a 2020 updated review. Gen Hosp Psychiatry. 2020;66:70-80. doi:10.1016/j. genhosppsych.2020.06.011

5. Guo J, Wang J, Sun W, et al. The advances of post-stroke depression: 2021 update. J Neurol. 2021:1-14. doi:10.1007/s00415-021-10597-4

6. Paolucci S. Advances in antidepressants for treating post-stroke depression. Expert Opin Pharmacother. 2017;18(10):1011-1017. doi:10.1080/14656566.2017.1334765

7. Rocca MA, Barkhof F, De Luca J, et al. The hippocampus in multiple sclerosis. Lancet Neurol. 2018;17(10):918-926. doi:10.1016/S14744422(18)30309-0

8. Wen H, Weymann KB, Wood L, et al. Inflammatory signaling in post-stroke fatigue and depression. Eur Neurol. 2018;80(34):138-148. doi:10.1159/000494988

9. Nakagawa Y, Chiba K. Role of microglial $\mathrm{m} 1 / \mathrm{m} 2$ polarization in relapse and remission of psychiatric disorders and diseases. Pharmaceuticals. 2014;7(12):1028-1048. doi:10.3390/ph7121028

10. Wang Y, Li M, Liang Y, et al. Chinese herbal medicine for the treatment of depression: applications, efficacies and mechanisms. Curr Pharm Des. 2017;23(34):5180-5190. doi:10.2174/ 1381612823666170918120018 
11. Gill BS, Kumar S. Antioxidant potential of ganoderic acid in Notch-1 protein in neuroblastoma. Mol Cell Biochem. 2019;456(1-2):1-14. doi:10.1007/s11010-018-3485-7

12. Jia Y, Zhang D, Li H, et al. Activation of FXR by ganoderic acid A promotes remyelination in multiple sclerosis via anti-inflammation and regeneration mechanism. Biochem Pharmacol. 2021;185:114422. doi:10.1016/j.bcp.2021.114422

13. Yang Y, Zhou H, Liu W, et al. Ganoderic acid A exerts antitumor activity against MDA-MB-231 human breast cancer cells by inhibiting the Janus kinase 2/signal transducer and activator of transcription 3 signaling pathway. Oncol Lett. 2018;16(5):6515-6521. doi:10.3892/ol.2018.9475

14. Cao FR, Xiao BX, Wang LS, et al. Plasma and brain pharmacokinetics of ganoderic acid $\mathrm{A}$ in rats determined by a developed UFLC-MS/MS method. J Chromatogr B Analyt Technol Biomed Life Sci. 2017;1052:19-26. doi:10.1016/j.jchromb.2017.03.009

15. Yu ZR, Jia WH, Liu C, et al. Ganoderic acid A protects neural cells against NO stress injury in vitro via stimulating beta adrenergic receptors. Acta Pharmacol Sin. 2020;41(4):516-522. doi:10.1038/ s41401-020-0356-z

16. Jiang ZM, Qiu HB, Wang SQ, et al. Ganoderic acid A potentiates the antioxidant effect and protection of mitochondrial membranes and reduces the apoptosis rate in primary hippocampal neurons in magnesium free medium. Pharmazie. 2018;73(2):87-91. doi:10.1691/ ph.2018.7108

17. Laing RJ, Jakubowski J, Laing RW. Middle cerebral artery occlusion without craniectomy in rats. Which method works best? Stroke. 1993;24(2):294-297. doi:10.1161/01.str.24.2.294

18. National Research Council (US) Committee for the Update of the Guide for the Care and Use of Laboratory Animals. Guide for the Care and Use of Laboratory Animals. 8th ed. Washington (DC): National Academies Press (US); 2011. doi:10.17226/12910

19. Hu MZ, Wang AR, Zhao ZY, et al. Antidepressant-like effects of paeoniflorin on post-stroke depression in a rat model. Neurol Res. 2019;41(5):446-455. doi:10.1080/01616412.2019.1576361

20. Song Q, Fan C, Wang P, et al. Hippocampal CA1 betaCaMKII mediates neuroinflammatory responses via COX-2/PGE2 signaling pathways in depression. $J$ Neuroinflammation. 2018;15(1):338. doi:10.1186/s12974-018-1377-0

21. Das J, Kr G. Post stroke depression: the sequelae of cerebral stroke. Neurosci Biobehav Rev. 2018;90:104-114. doi:10.1016/j. neubiorev.2018.04.005

22. Tu WJ, Qiu HC, Liu Q, et al. Decreased level of irisin, a skeletal muscle cell-derived myokine, is associated with post-stroke depression in the ischemic stroke population. J Neuroinflammation. 2018;15 (1):133. doi:10.1186/s12974-018-1177-6

23. Li C, Huang J, Cheng YC, et al. Traditional Chinese medicine in depression treatment: from molecules to systems. Front Pharmacol. 2020;11:586. doi:10.3389/fphar.2020.00586

24. Mondal AC, Fatima M. Direct and indirect evidences of BDNF and NGF as key modulators in depression: role of antidepressants treatment. Int J Neurosci. 2019;129(3):283-296. doi:10.1080/ 00207454.2018.1527328

25. Filho $\mathrm{CB}$, Jesse $\mathrm{CR}$, Donato $\mathrm{F}$, et al. Chronic unpredictable mild stress decreases BDNF and NGF levels and $\mathrm{Na}(+), \mathrm{K}(+)$-ATPase activity in the hippocampus and prefrontal cortex of mice: antidepressant effect of chrysin. Neuroscience. 2015;289:367-380. doi:10.1016/j.neuroscience.2014.12.048

26. Hong YP, Lee HC, Kim HT. Treadmill exercise after social isolation increases the levels of NGF, BDNF, and synapsin I to induce survival of neurons in the hippocampus, and improves depression-like behavior. J Exerc Nutr Biochem. 2015;19(1):11-18. doi:10.5717/ jenb.2015.19.1.11
27. Li W, Ling S, Yang Y, et al. Systematic hypothesis for post-stroke depression caused inflammation and neurotransmission and resultant on possible treatments. Neuro Endocrinol Lett. 2014;35(2):104-109.

28. Qin X, Wang W, Wu H, et al. PPARgamma-mediated microglial activation phenotype is involved in depressive-like behaviors and neuroinflammation in stressed $\mathrm{C} 57 \mathrm{BL} / 6 \mathrm{~J}$ and ob/ob mice. Psychoneuroendocrinology. 2020;117:104674. doi:10.1016/j. psyneuen.2020.104674

29. Wan B, Li Y, Sun S, et al. Ganoderic acid A attenuates lipopolysaccharide-induced lung injury in mice. Biosci Rep. 2019;39(5). doi:10.1042/BSR20190301

30. Cao T, Tang C, Xue L, et al. Protective effect of Ganoderic acid A on adjuvant-induced arthritis. Immunol Lett. 2020;226:1-6. doi:10.1016/ j.imlet.2020.06.010

31. Xu L, He D, Bai Y. Microglia-mediated inflammation and neurodegenerative disease. Mol Neurobiol. 2016;53(10):6709-6715. doi:10.1007/s12035-015-9593-4

32. Xue Y, Nie D, Wang LJ, et al. Microglial polarization: novel therapeutic strategy against ischemic stroke. Aging Dis. 2021;12 (2):466-479. doi:10.14336/ad.2020.0701

33. He T, Li W, Song Y, et al. Sestrin2 regulates microglia polarization through mTOR-mediated autophagic flux to attenuate inflammation during experimental brain ischemia. J Neuroinflammation. 2020;17 (1):1-13. DOI:10.1186/s12974-020-01987-y

34. Kalkman HO, Feuerbach D. Antidepressant therapies inhibit inflammation and microglial M1-polarization. Pharmacol Ther. 2016;163:82-93. doi:10.1016/j.pharmthera.2016.04.001

35. Zhang J, Xie X, Tang M, et al. Salvianolic acid B promotes microglial M2-polarization and rescues neurogenesis in stress-exposed mice. Brain Behav Immun. 2017;66:111-124. doi:10.1016/j. bbi.2017.07.012

36. Cheng Y, Xie P. Ganoderic acid A holds promising cytotoxicity on human glioblastoma mediated by incurring apoptosis and autophagy and inactivating PI3K/AKT signaling pathway. J Biochem Mol Toxicol. 2019;33(11):e22392. doi:10.1002/jbt.22392

37. Wang JQ, Mao L. The ERK pathway: molecular mechanisms and treatment of depression. Mol Neurobiol. 2019;56(9):6197-6205. doi:10.1007/s12035-019-1524-3

38. Li J, Luo Y, Zhang R, et al. Neuropeptide trefoil factor 3 reverses depressive-like behaviors by activation of BDNF-ERK-CREB signaling in olfactory bulbectomized rats. Int $J$ Mol Sci. 2015;16 (12):28386-28400. doi:10.3390/ijms161226105

39. Lu J, Zhou H, Meng D, et al. Tanshinone IIA improves depression-like behavior in mice by activating the ERK-CREBBDNF signaling pathway. Neuroscience. 2020;430:1-11. doi:10.1016/j.neuroscience.2020.01.026

40. Lu J, Liang J, Wang JR, et al. Acupuncture activates ERK-CREB pathway in rats exposed to chronic unpredictable mild stress. Evid Based Complement Alternat Med. 2013;2013:469765. doi:10.1155/ 2013/469765

41. Luo L, Deng S, Yi J, et al. Buyang huanwu decoction ameliorates poststroke depression via promoting neurotrophic pathway mediated neuroprotection and neurogenesis. Evid Based Complement Alternat Med. 2017;2017:4072658. doi:10.1155/2017/4072658

42. Li T, Wang D, Zhao B, et al. Xingnao jieyu decoction ameliorates poststroke depression through the BDNF/ERK/CREB pathway in rats. Evid Based Complement Alternat Med. 2018;2018:5403045. doi:10.1155/2018/5403045 


\section{Publish your work in this journal}

Neuropsychiatric Disease and Treatment is an international, peerreviewed journal of clinical therapeutics and pharmacology focusing on concise rapid reporting of clinical or pre-clinical studies on a range of neuropsychiatric and neurological disorders. This journal is indexed on PubMed Central, the 'PsycINFO' database and CAS, and is the official journal of The International Neuropsychiatric Association (INA). The manuscript management system is completely online and includes a very quick and fair peer-review system, which is all easy to use. Visit http://www.dovepress.com/testimonials.php to read real quotes from published authors.

Submit your manuscript here: https://www.dovepress.com/neuropsychiatric-disease-and-treatment-journal 